\title{
Los excluidos laboralmente por su condición de discapacidad: Egresados de la Fundación CE Camilo del Distrito de Barranquilla. ${ }^{1}$
}

\section{Laborally excluded by their disability condition: graduated from the Fundación CE Camilo del Distrito de Barranquilla}

\author{
Yariceth Ortiz de la $\mathrm{Hoz}^{2}$ \\ Rafael Alberto Zambrano Vanegas ${ }^{3}$ \\ RESUMEN
}

El presente trabajo contiene los resultados de la investigación titulada "Exclusión laboral de personas en situación de discapacidad egresados de la Fundación CE Camilo en el periodo comprendido del 2014 al 2018" que se refiere al problema social que afecta a ciertos grupos de personas. La exclusión laboral es un ámbito de este problema social que influye en la vida laboral de las personas, en este caso se investigó o indago como afecta a los egresados en condición de discapacidad de la Fundación Centro Educativo de Habilitación y Rehabilitación Integral San Camilo (CE CAMILO) y así también a su familia, entendiendo que el hecho de tener un integrante de la familia en condición de discapacidad genera situaciones y cambios con los que la familia tendrá que enfrentarse y acostumbrarse.

\section{Palabras claves:}

Exclusión laboral, dependencia, conflictos familiares, apego emocional, discapacidad.

\footnotetext{
${ }^{1}$ Este artículo es producto de la investigación realizada entre los años 2018 y 2019 en la ciudad de Barranquilla denominada "Exclusión laboral de personas en situación de discapacidad egresados de la Fundación CE Camilo en el periodo comprendido entre el 2014 al 2018".

2 Egresada del Programa de Trabajo Social Corporación Universitaria Rafael Núñez Sede Barranquilla. yortizd26@curnvirtual.edu.co

3 Docente del Programa de Trabajo Social Corporación Universitaria Rafael Núñez Sede Barranquilla. rafael.zambrano@curnvirtual.edu.co
}

Recibido: 25 de septiembre de 2019. Aprobado: 15 de noviembre de 2019. 


\begin{abstract}
This work contains the results of the research entitled "Labor exclusion of people with disabilities graduated from the CE Camilo Foundation in the period from 2014 to 2018" that refers to the social problem that affects certain groups of people. Labor exclusion is an area of this social problem that influences the working life of people, in this case it was investigated or investigated how it affects graduates in disability condition of the San Camilo Integral Habilitation and Rehabilitation Educational Center Foundation (CE CAMILO) and also his family, understanding that the fact of having a family member in a condition of disability generates situations and changes that the family will have to face and get used to.
\end{abstract}

\title{
Key words:
}

Work Exclusion, Dependency, Family Conflicts, Emotional Attachment, Disability.

\section{Introducción.}

El informe que se presenta a continuación, refiere los resultados del proceso de la investigación desarrollada durante el segundo ciclo del 2018 y el primer ciclo del 2019 en la

Fundación CE CAMILO. La investigación pretende identificar las afectaciones que presentan las personas en situación de discapacidad y sus familias.

Alcanzar un espacio laboral puede ser fácil para aquellas personas que tienen las condiciones físicas, cognitivas y técnicas, pero para las personas en situación de discapacidad esto puede ser una tarea difícil ya que por sus condiciones sus posibilidades se reducen. En Colombia hay estadísticas desiguales en cuanto al ingreso a un empleo, según el DANE hay 3.5 millones de personas que tienen alguna limitación ya sea física, cognitiva, sensorial y mental y solo el $39.3 \%$ de esa población está laborando, de igual forma en el departamento del Atlántico, se cuenta con una política de discapacidad de la cual de 14.000 personas en situación de discapacidad solo 180 laboran actualmente. Teniendo en cuenta esta situación es preciso decir que se está evidenciando una exclusión laboral que no solo afecta a las personas con tal limitación, también afecta su desarrollo personal, su familia y su entorno. 
En la ciudad de Barranquilla actualmente se cuenta con la Política Pública de Discapacidad; esta empezó a funcionar en 2016 pero no se está efectuando como debería o no se están realizando las acciones pertinentes para que se vean resultados positivos, ya que hasta el 2018, solo 180 personas en situación de discapacidad se encuentran laborando. En este sentido, hay una población mayor que no está siendo beneficiada por esta política, entre esas los egresados de la Fundación CE Camilo. Mediante una serie de técnicas e instrumentos de recolección de información como lo son la encuesta y la entrevista al igual que la realización de una observación directa, propios del Trabajo Social y de la investigación. Se indagó sobre las posibles causas que evidencian este problema social como lo es la exclusión laboral y la manera cómo afecta a una serie de sistemas, afirmando de acuerdo a los instrumentos aplicados que si hay una afectación a nivel individual y familiar, como lo son los bajos recursos económicos, los cuidados que requiere una persona en situación en discapacidad, esto teniendo en cuenta el tipo de discapacidad que tenga.

A los bajos recursos económicos que poseen las familias de estas personas se suman otros factores que desencadenan situaciones de pobreza, estrés y conflictos familiares en los cuales el más afectado es la persona en situación de discapacidad. (De León, Ballestas y Martínez, 2017)

Este proyecto de investigación se inscribe en la Línea de Investigación de: Trabajo Social y Derechos Humanos del programa de Trabajo Social de la Corporación Universitaria Rafael Núñez Campus Barranquilla.

\section{Diseño Metodológico}

Con el fin de identificar las afectaciones que presentan los egresados en situación de discapacidad de la Fundación CE CAMILO y sus familias, se empleó el tipo de estudio mixto

(cuantitativo y cualitativo) de naturaleza descriptivo con un enfoque mixto (Inductivo y deductivo) y el tipo de muestreo es intencional ya que se observó detalladamente las 
situaciones y se contrastó la información para establecer conclusiones que permiten consolidar y verificar dicha información.

De los 47 egresados sólo se aplicaron los instrumentos a 20 de ellos (muestra intencional), a quienes va dirigida la investigación, egresados de la Fundación Centro Educativo de Habilitación Rehabilitación Integral San Camilo entre los años 2014 a 2018, con edades comprendidas entre 18 y 22 años que presentaron alguna discapacidad ya sea física o cognitiva, representados por 4 mujeres y 16 hombres. También se le aplicó una entrevista al familiar cuidador, responsable de cada uno de los integrantes del grupo de egresados.

Se aplicaron a los egresados en situación de discapacidad encuestas dirigidas a determinar si en realidad se está evidenciando exclusión, al igual que las afectaciones derivadas de este problema social hacia ellos y sus familiares. De igual manera se aplicaron entrevistas a los familiares cuidadores, para conocer cómo los afecta esta situación, esto desde distintos ámbitos: económico, social y familiar.

\section{Resultados}

La aplicación de los instrumentos se llevó a cabo en la Fundación CE CAMILO. Se presentaron 20 de los 45 egresados del 2014 al 2018. Con cada uno de sus familiares cuidadores, se realizó una charla sobre emprendimiento puesto que la entidad (la Fundación) pretende crear programas sobre inclusión laboral que permita el acceso a la vida laboral de los egresados. Se aplicó una encuesta a egresados para recolectar información que permitiese analizar los obstáculos que pueden encontrar y que le imposibilitan la inserción laboral de estas personas; así también, las afectaciones derivadas de esta problemática social que los afecta a ellos y sus familiares. Debido a su discapacidad cognitiva se dificultó la aplicación del instrumento, pero se concluyó exitosamente. 


\section{Inserción laboral}

Desde una perspectiva de inserción laboral con los egresado de la Fundación CE Camilo, sobre todo con la percepción que ellos tienen en cuanto a los estereotipos que los potenciales empleadores o las personas a las cuales recurrieron solicitando una oportunidad de trabajo, tienen en cuanto a la prevención sobre las limitaciones que tienen estas personas con cualquier tipo de discapacidad: las respuestas (en porcentajes) de los egresados de la Fundación se orientan en el sentido de recibir discriminación, tal como se aprecia en la Gráfica siguiente.

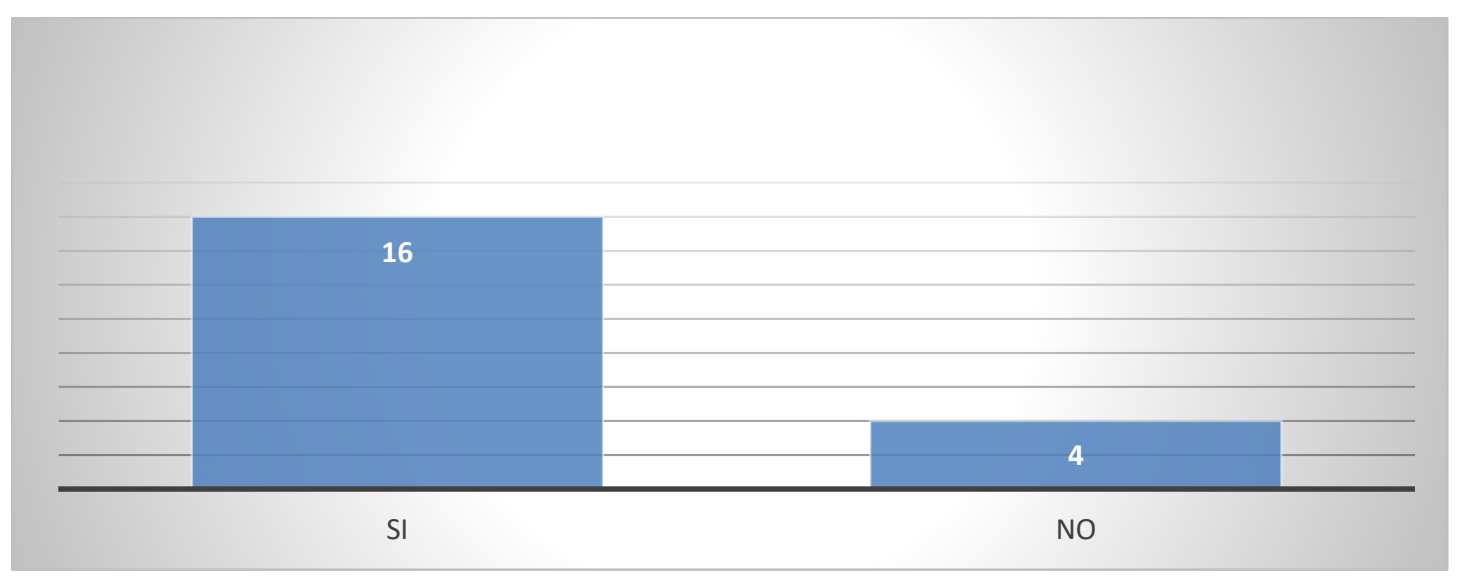

Grafica 1. Estereotipos de personas (potenciales empleadores) frente a discapacitados. Fuente: Elaboración propia a partir de los datos de la encuesta.

De los 20 egresados de la Fundación CE Camilo a quienes se les aplicó una encuesta, 16 de ellos aseguran que si recibe comentarios estereotipados; también se han sentido estigmatizados, puesto que es muy frecuente que sean tildado de incapacitados, incluso tratados como niños siendo mayores de edad, de esta manera lo expresaron ellos y sus familiares, los cuales manifiestan que estos comentarios 0 acciones son muy frecuentes en el entorno en el que conviven, siendo las personas desconocidas o los amigos quienes frecuentan este tipo de actos o acciones que en muchas ocasiones son ofensivas para las personas afectadas. Aunque no parezcan mal 
intencionados, afecta el individuo que tiene una limitación, al igual que a su familia. Contrasta esta percepción con la registrada por cuatro personas que manifestaron no haber sido discriminadas por su situación o no manifestaron percibir comentarios ofensivos.

Por otra parte, la Fundación CE Camilo a estas personas (egresados de la entidad) les otorga la posibilidad de realizar un curso técnico para que puedan desempeñarse en la vida laboral, en actividades orientadas o enfocadas a la modistería, carpintería 0 panadería. Estas acciones se realizan a través de convenios entre la institución y el Servicio Nacional de Aprendizaje SENA, quien le brinda las clases y la certificación a cada uno de los asistentes, lo cual implica que todos los egresados cuentan con un título técnico. Sin embargo, pese al título, no se les ha facilitado el poder obtener un empleo.

Entre los rasgos diferenciadores que distinguen al grupo de los egresados de la Fundación CE Camilo con otros grupos que tengan cierta discapacidad, se pueden encontrar aspectos ligados a la procedencia de estos y a situaciones que hacen que se marquen los estereotipos por parte de los posibles empleadores, como había sido descrito por los investigadores McCauley, Stitt y Segal (1980), retomado posteriormente por Chacón Fuertes (1986) estableciendo los siguiente: "los estereotipos serían realmente "predicciones probabilísticas", lo que contribuye a que se tengan ciertas prevenciones (características diferenciales) en cuanto a las capacidades del grupo y su pertenencia a una determinada institución.

De acuerdo a lo que se manifestado como definición de los estereotipos y teniendo en cuenta las acciones de las personas y lo expresado por los encuestados, se puede decir que a nivel general hay un concepto erróneo de estos, por cuanto estas personas toman este concepto en forma negativa y como una opción para ofender, el hecho de ser diferentes no los cohíbe de un derecho fundamental y es el respeto, derecho que también es vulnerado a través de las ofensas por los estereotipos.

Al realizar la comparación entre las situaciones de rechazo o discriminación que algunas empresas hacen cuando se trata de una persona con limitaciones especiales con la condición laboral que actualmente tienen los egresados de la Fundación CE 
Camilo, se encuentran aspectos como los que se muestran en las siguientes Gráficas 2 y 3:

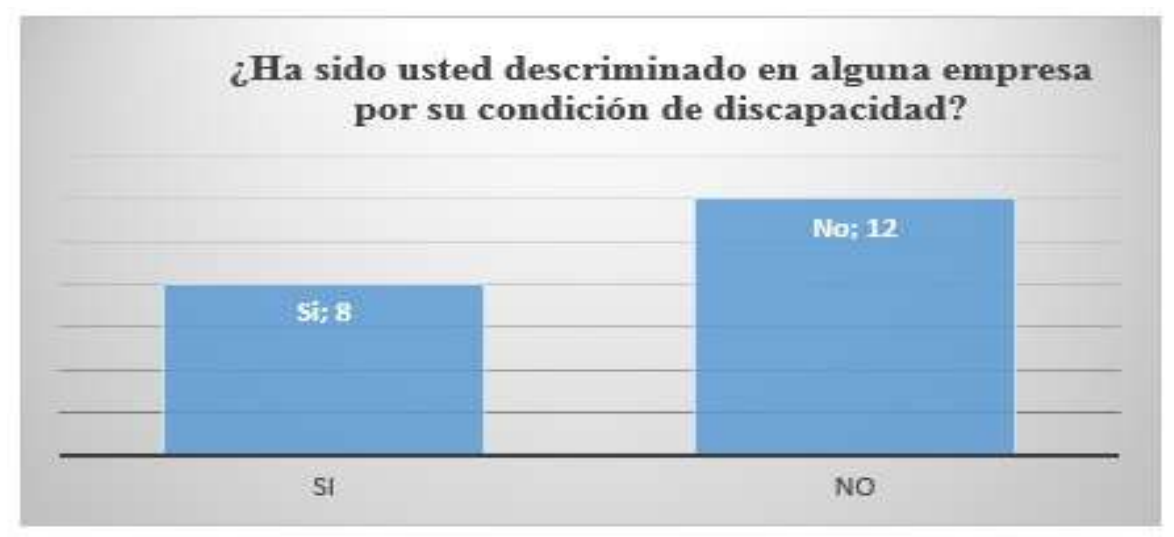

Grafica 2. Discriminación por tener una condición especial

Fuente: Elaboración propia a partir de los datos de la encuesta.

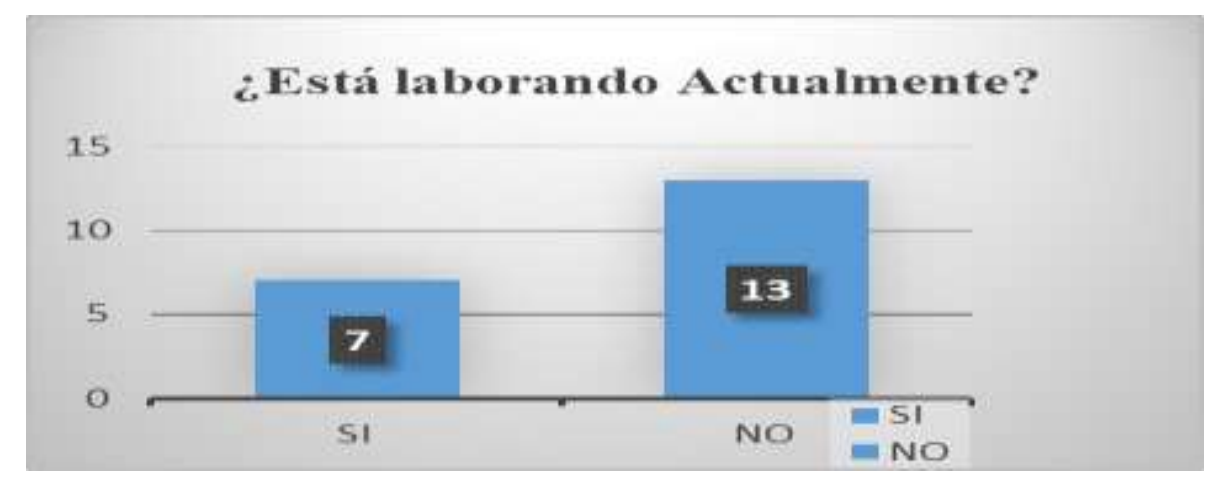

Gráfica 3. Dedicación actual de los egresados de la Fundación CE Camilo.

Fuente: Elaboración propia a partir de los datos de la encuesta.

Por un lado, mientras un 40\% (8 personas) de los egresados encuestados manifiesta haber sido discriminado por su condición de discapacidad, el 60\% (12 personas) niega esta información en forma rotunda, pero ese mismo porcentaje no se refleja en las condiciones laborales actuales, ya que sólo están trabajando el 35\% (7 personas) y permanece sin ocupación el $65 \%$ (13 personas). 
Aunque Colombia estableció la Ley 361 de 7 de febrero de 1997, la cual establece en el Artículo $2^{\circ}$ que "El Estado garantizará y velará por que en su ordenamiento jurídico no prevalezca discriminación sobre habitante alguno en su territorio, por circunstancias personales, económicas, físicas, fisiológicas, síquicas, sensoriales y sociales" (Congreso de Colombia, p. 1), creando los mecanismos de integración para que las personas con limitación, establezcan entre ellas la promoción del autoempleo y la creación de empresas por parte de las personas con discapacidad, es común encontrar que estas personas laboren en la informalidad, porque en el fondo le temen al rechazo y a la discriminación por sus condiciones diferentes.

El Registro para la Localización y Caracterización de las Personas con Discapacidad (RLCPD) en Colombia, revela que de las personas con discapacidad que se encuentran ocupada en cualquier actividad, el $80 \%$ lo hace sin tener un contrato laboral, lo que se traduce en informalidad, razón por la cual, en la mayoría de los casos estas personas no gozan de seguridad social, ni cotizan en salud ni para la pensión.

Por ello ante las dos preguntas formuladas en la encuesta en el sentido de haber sido discriminado o si han tenido una percepción indicadora de tal situación, por parte de alguna empresa, la mayor parte de los egresados manifestó no haber sentido esto, pero no se encuentran laborando. Es un aspecto que en apariencia no guarda sintonía con lo que es visible en la Gráfica 3, si tiene en cuenta que la discapacidad que los egresados tienen es cognitiva, se puede decir que como tal ellos no han tenido la capacidad de sentir tal discriminación. Son los padres de estas personas, en una entrevista realizada con ellos quienes afirman que si los han discriminados, al igual que la misma Fundación CE Camilo, quien hace gestión en forma diaria en la búsqueda de convenios o elaborando propuestas en pro de crear programas con entidades que les permita a sus egresados tener el acceso al empleo.

Esta información relacionada con las actividades y la gestión de la Fundación es suministrada a los padres o familiares cuidadores quienes tienen la responsabilidad de los egresados, y además, son los encargados de otorgarles el permiso o la libertad de trabajar, ya que algunos padres han optado por no permitir que sus hijos sean discriminados. Situación que los ha conducido a parecer con el rol de padres 
sobreprotectores, lo cual ha conducido a una especie de dependencia de hijos o egresados hacia sus familiares.

Rodríguez Díaz \& Ferreira (2010) conceptúan una definición de la discapacidad, siguiendo los parámetros expuestos por Canguilhem (1970) quien propone que lo normal del concepto se muestra como algo "dinámico y polémico. La normalidad puede entenderse de dos maneras. Por un lado, lo normal es aquello que es tal como debe ser; por otro lado, lo normal es aquello que se encuentra en la mayoría de los casos" (p. 160) y que a los egresados de la Fundación CE Camilo. Se les muestra como un obstáculo que de acuerdo con la concepción de las otras personas representa un conjunto de condiciones materiales y sociales que contribuyen de manera acumulativa a la marginación, incapacitación y exclusión de las personas con cierta discapacidad.

De acuerdo con lo anterior se puede decir que las condiciones sociales influyen más en la marginación de las personas, puesto que estas se sienten con toda la voluntad de sobrepasar las condiciones materiales, pero lamentablemente, los egresados de la Fundación CE Camilo no alcanzan siquiera a aproximarse a esto, ya que el rechazo, la sobreprotección, la discriminación y la falta de apoyo han hecho a estas personas vivir en marginación no solo material si no también emocional. Su familia no es una marginación absoluta ni grave ya que la unidad familiar ha optado por no permitir esta situación, que los egresados no alcanzan a percibir.

De los 20 usuarios (egresados de la Fundación) apenas siete de ellos se encuentran laborando de manera informal en sus casas o en negocios cercanos a estas. El ejercicio de estos es la venta de un refresco preparado con frutas naturales denominado cotidianamente "bolis", pescado, venta de minutos por celular (llamadas telefónicas a móviles) o en salones de belleza, que son negocios establecidos por sus padres y bajo supervisión de ellos, o por particulares conocidos, con la intención de que estas personas no se sientan inoperantes. El padre de familia de los egresados encuentra en estas actividades una forma para brindarle seguridad y la protección de ellos y no arriesgarlos, puesto que un empleo implica características específicas como responsabilidades y funciones exactas además el tener que transportarse y movilizarse hasta su lugar de trabajo. Esta situación de tener alejados a los padres o familiares 
cuidadores representa un riesgo que tanto ellos como sus hijos corren. El trabajo o el desarrollo de actividades en su propio hogar, representa para los egresados la seguridad y el evitar peligros, ya que no están en la necesidad de transportarse.

Ante la pregunta consignada en la encuesta y relacionada con los aspectos anteriormente descritos, todos los egresados afirmaron que se les facilita desplazarse hasta su lugar de trabajo, y consideraron que la infraestructura de las viviendas donde viven o desarrollan sus actividades está acorde con sus necesidades, como bien se puede observar en la Gráficas 4 y 5.

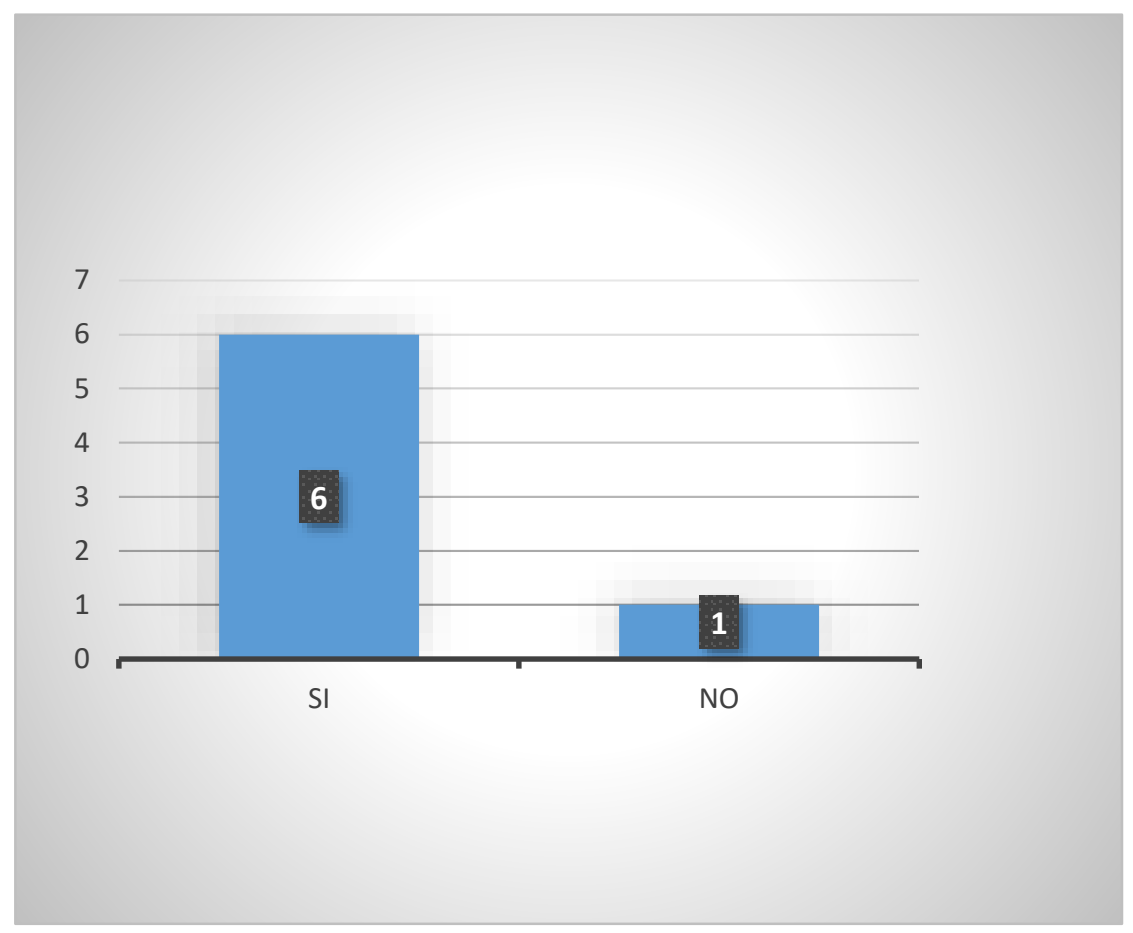

Grafica 4. Dificultad de comunicación.

Fuente: Elaboración propia a partir de los datos de la encuesta. 


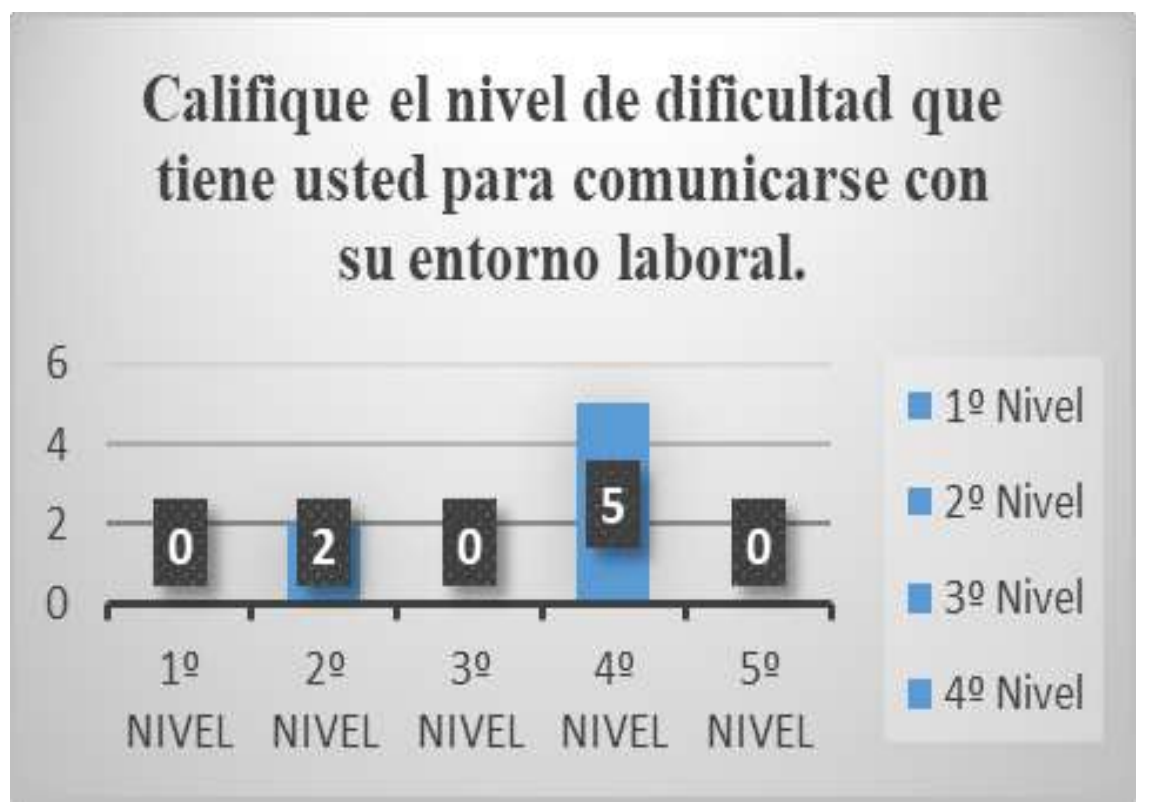

Gráfica 5. Nivel de dificultad de los egresados de la Fundación CE Camilo.

Fuente: Elaboración propia a partir de los datos de la encuesta.

Los siete egresados que se encuentran laborando todos tienen una discapacidad cognitiva, y afirman que se les dificulta la comunicación con su entorno familiar y laboral. Generalmente, los problemas más frecuentes de lenguaje se producen en el ámbito de la articulación y pronunciación, trastornos de la voz y tartamudez. Aun así, las alteraciones de lenguaje son más frecuentes en los niveles severo y profundo, y al interior de ellas, como lo han expresado los investigadores Morodo y Martínez (2010) en sintonía como lo expuesto por Fernández Gabalón (2013) la más notorias son las de articulación, que tienen "alterados los procesos de control cognitivo, manifiestan problemas muy 
evidentes en la generalización de los aprendizajes que adquieren y, como consecuencia de todo ello, van a tener dificultades permanentes en su proceso de aprendizaje" (p. 31).

Esta situación se presenta, debido a la naturaleza de la discapacidad lo cual es evidente por las dificultades que los egresados tienen con la comunicación que, además de dificultosa y limitada, se le agrega el hecho de estar recibiendo constantemente comentarios ofensivos y burlas por su forma de hablar. Con el propósito de evitar el sarcasmo y los ironías, la mayoría opta por limitar o evitar la comunicación lo más que se y solo expresar lo necesario.

En Colombia, algunas instituciones como en el caso de la Fundación Best Buddies Colombia y otras más, "muestran como el crecimiento empresarial ha estado acompañado de ejercicios de responsabilidad social empleando a personas con discapacidad cognitiva" (Álvarez Ladino, et.al. 2015, p. 43), con un programa como "Amigos del alma", en el que se evidencian acciones "de promoción y garantía de la vinculación laboral en pro de mantener y mejorar la calidad de vida brindando de esta manera una rentabilidad económica y social” (p. 44).

\section{Afectaciones socioeconómicas}

La mayor parte de los hogares de los egresados de la Fundación CE Camilo, pertenecen al estrato socioeconómico 1, exactamente 16 de ellos están adscritos a este nivel y solamente cuatro forman parte del estrato 2. Estos hogares se encuentran en los sectores pertenecientes a barrios del suroccidente del Distrito de Barranquilla cómo Las Malvinas, Carrizal, Me Quejo, Cuchilla Villate, en los que la inseguridad y el subempleo son el pan de cada día debido a la pobreza que se evidencia en estos sectores de la ciudad. Para algunas familias su estrato socioeconómico no es sinónimo de bajos ingresos económicos, y esto se evidencia en la Gráfica 5, en la que se observa que el $50 \%$ de los egresados manifiesta obtener los ingresos económicos suficientes para satisfacer sus necesidades, pero al mismo tiempo evidencian que su diario vivir es 
preocupante puesto que las necesidades diarias se satisfacen de acuerdo a los recursos obtenidos de los trabajos informales. Si bien es cierto, en todas las familias hay una persona que aporta ingresos económicos o que labora, estos no son suficientes, ya que los hogares colombianos tienen que responder por obligaciones que tiene a su cargo el Estado, las cuales constituyen las necesidades de todo ser humano, entre los cuales se encuentran los servicios públicos, alimentación, transporte, entre otra y que son propias de la sustentación y el desarrollo de cada ser humano y cada familia. A ello se agrega el dato sobre aquellas familias que no cuentan con casa propia, las cuales se ven obligadas a pagar arriendo, además de los gastos que una persona en condición de discapacidad representa.

En las Gráficas 6 y 7 se muestran las afectaciones que ligadas al estrato socioeconómico de las familias de los egresados y al ingreso que reciben en forma mensual se puede extraer conclusiones alrededor de la imposibilidad de satisfacer la necesidades básicas.

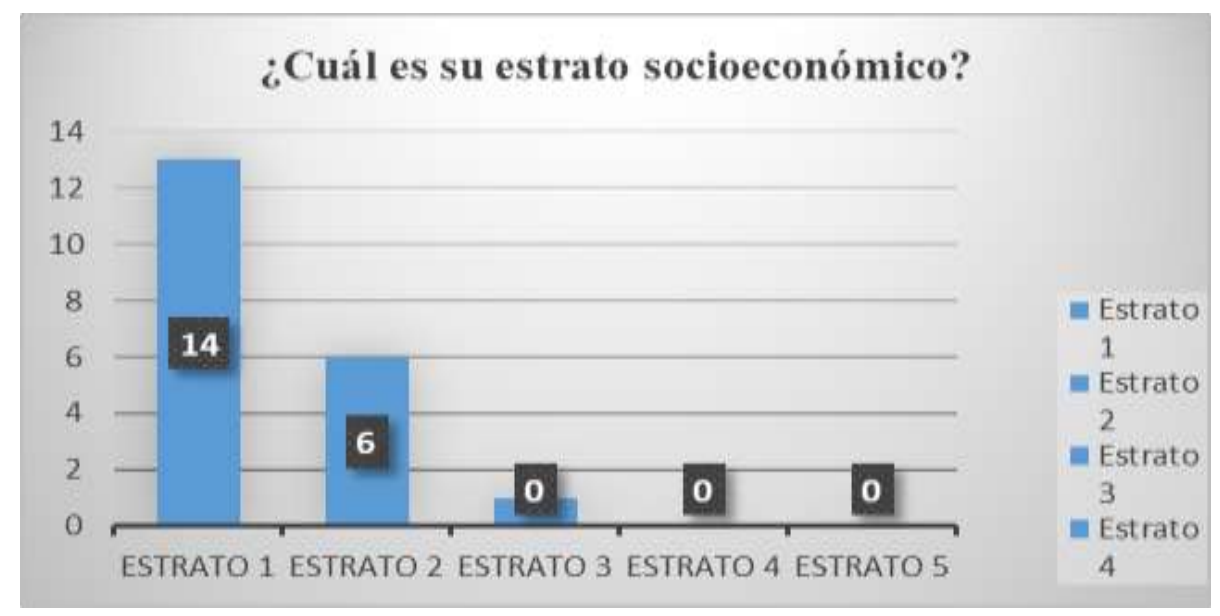

Gráfica 6. Los niveles socioeconómicos de las familias de los egresados de la Fundación CE Camilo.

Fuente: Elaboración propia a partir de los datos de la encuesta. 


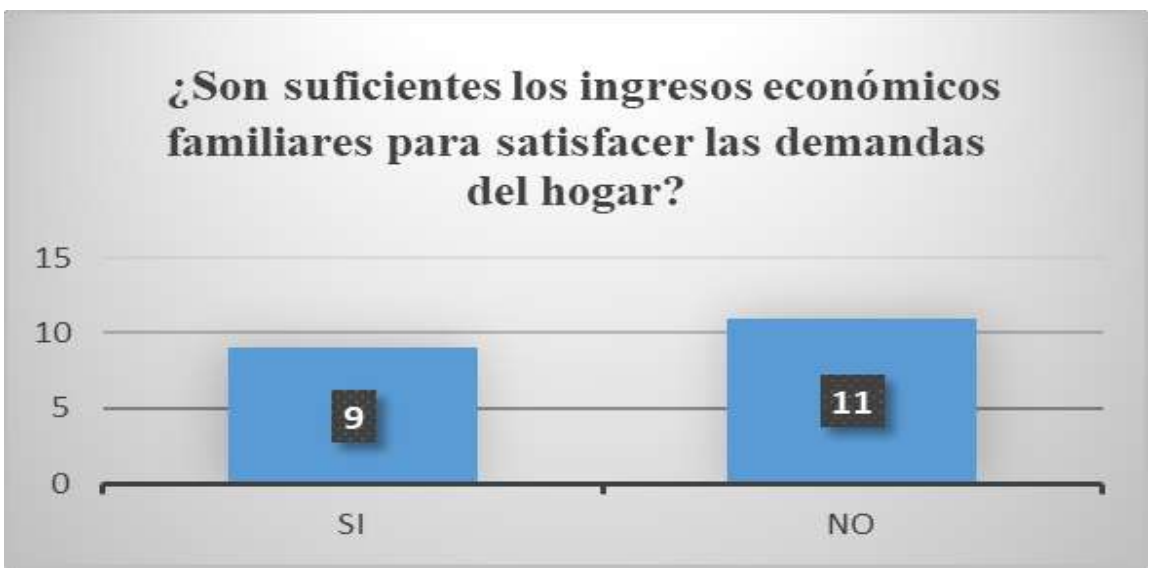

Grafica 7. Ingresos económicos y satisfacción de necesidades básicas

Fuente: Elaboración propia a partir de los datos de la encuesta.

Co se puede apreciar, sólo en nueve hogares en donde habita más de una persona que aporta ingresos y la familia es extensa, se evidencia una aparente satisfacción de las necesidades básicas. En la mayoría de las familias (11), la persona encargada de los cuidados del sujeto afectado por una condición de discapacidad es su madre o los hermanos con un nivel de responsabilidad, ya que el padre es quien mayormente aporta al hogar los ingresos económicos o a las demandas de este, permanece ocupado en el desarrollo y cumplimiento de sus obligaciones laborales. En hogares monoparentales, que en su mayoría son conformados por madre y hermanos, la madre es la que aporta el único ingreso económico, siendo un agravante para la condición socioeconómica de una familia.

La independencia económica de las personas con cualquier tipo de condición de discapacidad ha sido tratada por investigadores de las Ciencias Sociales, entre las que se encuentran disciplinas con Sociología, Trabajo Social, Psicología y algunas de las Ciencias de la Salud: Prevención y manejo de la discapacidad para trabajar: un campo de estudios necesario para abordar los retos del sistema colombiano de riesgos laborales, Diana E. Cuervo-Díaz y Marisol Moreno-Angarita (2017); Estado del arte de la discapacidad en Colombia desde la normatividad y políticas públicas en salud durante el 
periodo comprendido entre el año 2000 y 2015 de la autoría de Deydy Johanna Dequia Rodríguez y María Del Mar Pazos Verdugo (2015); Aproximación a las capacidades de investigación en discapacidad en Colombia de las investigadoras Clara Duarte-Cuervo, Aleida Fernández-Moreno, Israel Cruz-Velandia y Solángel García-Ruiz (2015); Discapacidad y política pública: una apuesta política desde el discurso de niños y niñas, Karin Garzón Díaz (2014); Jóvenes con discapacidades: sujetos de reconocimiento de Carmen Aleida Fernández Moreno (2011); Abordaje de la discapacidad en facultades de Psicología de universidades privadas de Bogotá, entre 1998 y 2009, Mónica Lucía Castelblanco Niño (2011), entre otros trabajos.

En la Gráfica 8 se muestra los resultados de las respuestas suministradas por los egresados de la Fundación CE Camilo con respecto al nivel de independencia económica, y la forma como mes asumida por estos.

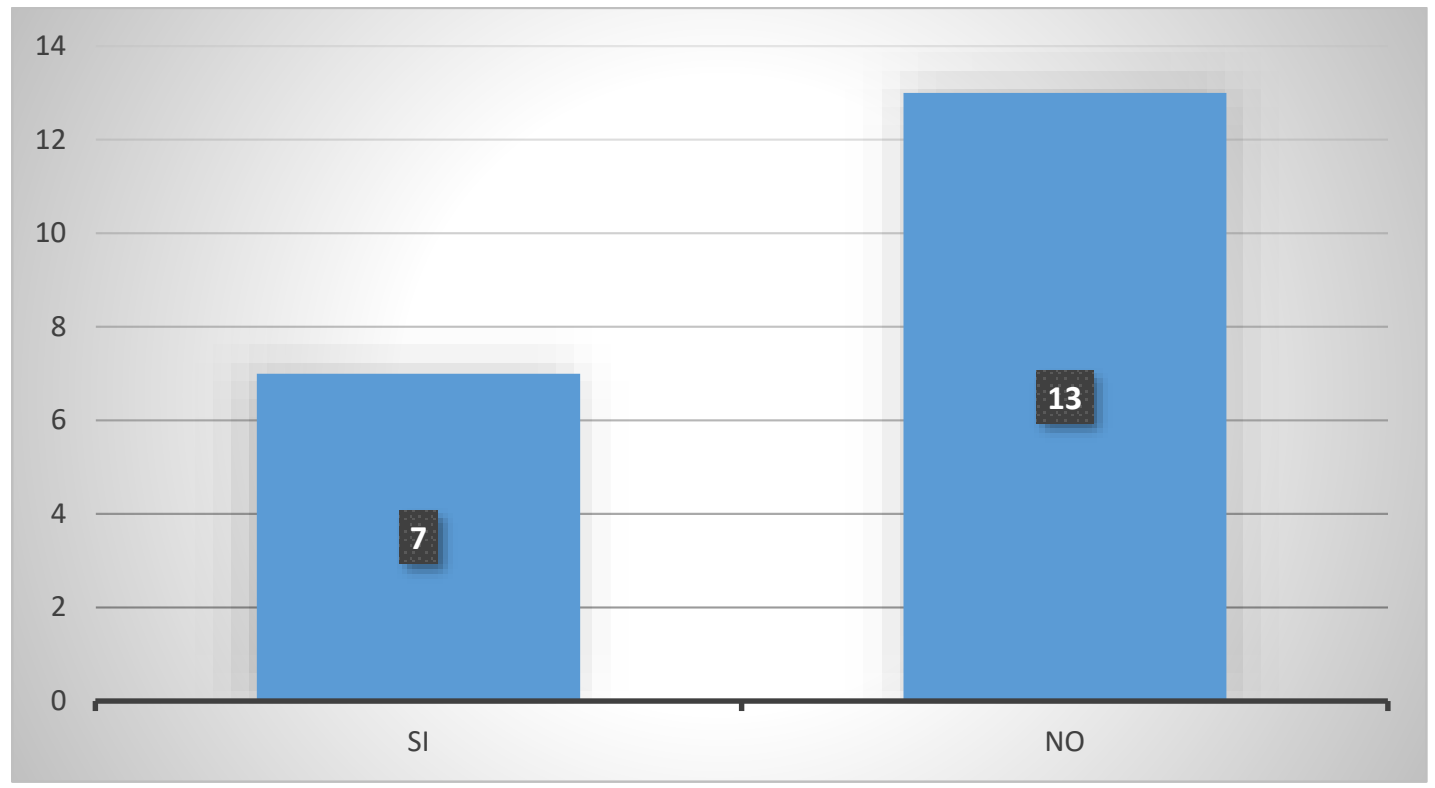

Grafica 8. Independencia económica de los egresados de la Fundación CE Camilo.

Fuente: Elaboración propia a partir de los datos de la encuesta.

La independencia económica es un factor importante en la vida de cada uno ya que de esto se evidencia la utilidad y la producción de cada persona, las personas necesitan estabilidad y esta se evidencia a través de su nivel económico, por esto es importante que los seres humanos y en especial las personas en situación de 
discapacidad se sientan independientes y puedan ser independientes tanto de sus actos como de sus responsabilidades y su estabilidad económica, tal como se alude en La Convención de las Naciones Unidas sobre los Derechos de las Personas con Discapacidad, celebrada en diciembre de 2006, que Padilla-Muñoz (2010) retoma en el artículo Discapacidad: contexto, concepto y modelos con el propósito de "promover, proteger y asegurar el goce pleno y en condiciones de igualdad de todos los derechos humanos y libertades fundamentales por todas las personas con discapacidad, y promover el respeto a su dignidad inherente, la autonomía individual, incluida la libertad de tomar las propias decisiones y la independencia de las personas” (p. 390).

Teniendo esto en cuenta lo anterior, en las respuestas de los egresados de la Fundación CE Camilo se resalta en la Gráfica que a continuación se presenta que 13 de ellos en situación de discapacidad no laboran y consideran que no son independientes económicamente. Contario a esto, los siete egresados que se encuentran laborando con empleos informales, consideran que son independientes económicamente. Sin embargo se tiene que indicar que sus ingresos provienen de la venta de "bolis", cubetas de jugo, pescado, dulces, entre otras cosas.

Las actividades que desarrollan los egresados son vigiladas por sus padres o en su defecto por el familiar cuidador; en realidad no reciben un ingreso económico fijo y su labor se basa solo en atender a los clientes. Esto quiere decir que, en realidad no disponen de independencia, más bien mantienen una dependencia en todos los aspectos de sus familiares, ya que los recursos que obtienen no son suficientes y por tal razón su familia tiene que sustentar cada una de sus necesidades, en este caso, de la persona que aporta la mayor parte de los ingresos que casi siempre es el padre. Ellos aportan a la economía familiar, sin embargo no tienen ingresos económicos por su empleo. Algunos de ellos reciben subsidios de las Cajas de Compensación, que equivale a un mínimo aporte que se hace al hogar. Cuando manifiestan sentir una satisfacción por su aporte, lo dicen porque aparentemente "no les falta nada porque nosotros padres cumplimos con 
todos los gastos, y ellos están convencidos que se debe a sus ingresos por la labor realizada"2.

\section{Conflictos familiares}

La Organización Mundial de la Salud (2011), presentó en el Informe mundial sobre la discapacidad 2011 datos relacionados con esta situación que no es exclusiva de un solo país y que permiten tomar decisiones acertadas en materia de políticas y programas sobre discapacidad, estableciendo que con la "información sobre el número de personas con discapacidad y sus circunstancias personales permite mejorar las medidas orientadas a eliminar las barreras discapacitantes y prestar servicios que promueven la participación de las personas con discapacidad" (p. 23). Los datos son preocupante hasta el extremo de considerar que las "personas con discapacidad están más expuestas al riesgo de sufrir violencia que las demás" $(p, 66)$. Sin embargo, se observa que en las familias de los egresados de la Fundación CE Camilo, por los datos registrado en la encuesta y en las entrevistas con los familiares, apenas en cinco casos se han manifestado brotes de violencia ocasionados por los bajos ingresos que perciben estos núcleos y que corresponden a quienes expusieron que se encuentran en términos de dependencia.

\footnotetext{
${ }^{2}$ De las entrevistas con los padres de los egresados de la Fundación CE Camilo o los familiares cuidadores. Barranquilla. octubre de 2019.
} 


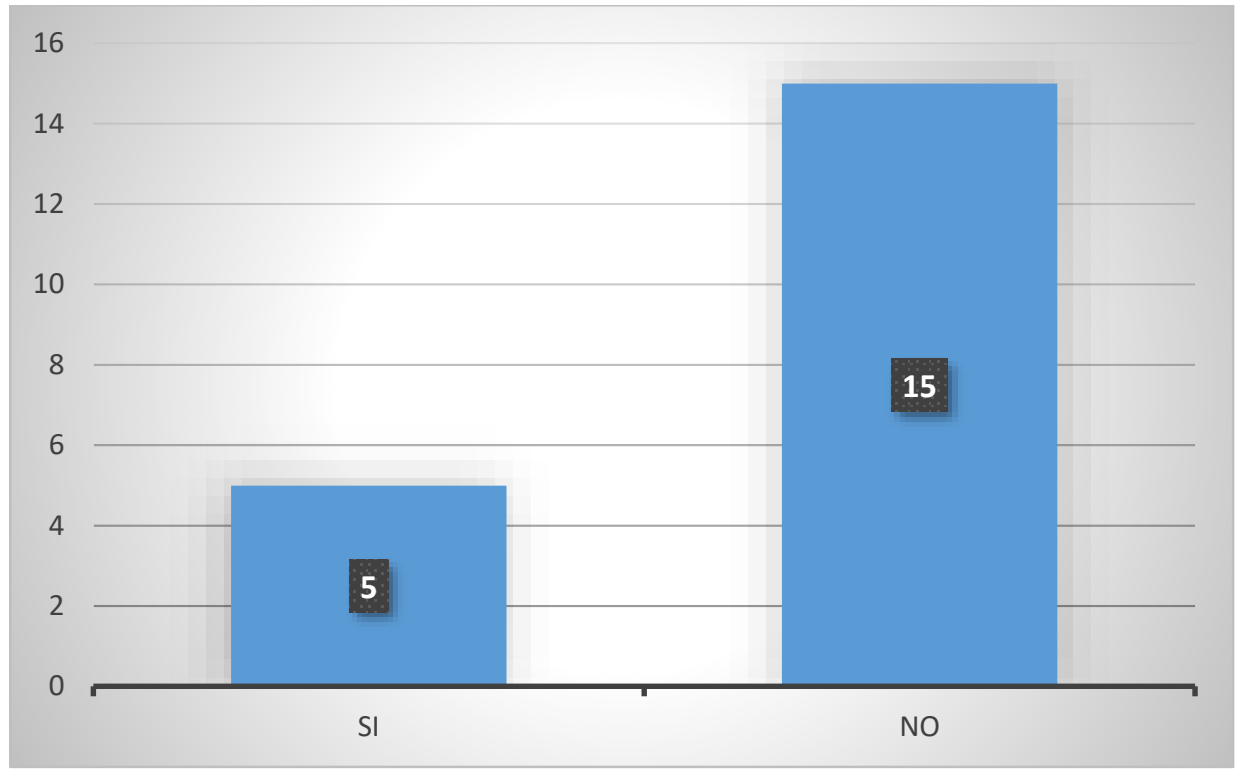

Grafica 2. Existencia de Conflictos familiares a causas de los bajos ingresos.

Fuente: Elaboración propia a partir de los datos de la encuesta.

También existe una variante de la situación y es el hecho que los familiares cuidadores afirman de manera categórica que por tener una persona en situación de discapacidad no cambia ni afecta la relación entre los integrantes de la familia, teniendo en cuenta que los 20 egresados presentan una discapacidad cognitiva, los familiares expresan que afrontaron la noticia de manera natural, con ciertos temores debido a la cultura del medio y en su conjunto de la sociedad que estigmatizan a personas con alguna situación fuera de lo normal, condenándola a exclusión, entre otras cosas.

De acuerdo con información emanada del Banco Interamericano de Desarrollo BID, al momento de presentarse alguna manifestación de incapacidad a una persona, la tendencia de la familia se orienta a empobrecerse en un 30\%. La explicación está apoyada en la consideración de que cuando dos personas del núcleo familiar dejan de trabajar (una porque es excluida del mercado laboral, y la otra porque, en muchos casos, decide abandonar su empleo) para cuidar a su pariente, ya no hay quien se responsabilice de la carga del hogar y el procurar para que los ingresos no falten. En la mayoría de los casos, el segundo actor es una mujer. Esta situación es más ocurrente en las familias de bajos niveles de ingreso. 
Es oportuno decir que no se pueden excluir las ocurrencias de afectaciones sociales y económicas, dado que una persona en situación de discapacidad, tiene los mismos gastos sin generar grandes aportes (cuando tiene alguna actividad). A ello se le adicionan los costos para atender citas médicas a las cuales no puede asistir sólo manifestado por los cuidadores- a los lugares alejados de su hogar, por lo que necesita un acompañantes. Estas son situaciones que contribuyen a generar conflictos familiares ocasionados por la exclusión laboral.

Se encontraron en la gran variedad de tipos de familia, aquellas que monoparentales en donde el padre o la madre son los únicos que aportan ingresos a la familia, además del desgaste laboral, económico y emocional que recibe esta persona, se amplía cuando tiene que dejar la responsabilidad a otro cuidador, como hermanos o abuelos.

Los datos obtenidos a través de la encuesta y la entrevista permiten afirmar que si hay exclusión laboral y que efectivamente las afectaciones derivadas de esta problemática social (afectaciones de tipo económico, social y familiar) son las que debe enfrentar y soportar cada uno de los egresados de la Fundación CE Camilo en situación de discapacidad.

Las afectaciones más notables de la exclusión laboral sin duda alguna está representada por la dificultad para obtener un empleo, ya que esto desencadena otras afectaciones como lo son, no tener ingresos económicos, el no poder aportar ingresos a su familia, no poder satisfacer sus necesidades básicas como todo ser humano y tiene que esperar que sean sus padres o sus familiares los encargados de resolverles esta situación.

De acuerdo con los planteamientos de Oliver (1998) se puede entender que es:

la sociedad la que incapacita físicamente a las personas con insuficiencias. La discapacidad es algo que se impone a nuestras insuficiencias por la forma en que se nos aísla y excluye innecesariamente de la participación plena en la sociedad. Por tanto, los discapacitados constituyen un grupo oprimido de la sociedad. La 
discapacidad no se relaciona con el cuerpo sino que es una consecuencia de la opresión social: la discapacidad es total y exclusivamente social" (p. 41).

Teniendo esto en cuenta se puede afirmar este argumento ya que dada las estadísticas e instrumentos aplicados, las personas en condición de discapacidad son vistas como cargas o como seres incapaces, por lo que, tanto sus familiares como la sociedad no dan libertad a sus acciones ni a su libre desarrollo. Las personas en situación de discapacidad tienen ciertos obstáculos o limitaciones sociales y familiares que no les da acceso a la inserción laboral, pero el estigma social desencadena todos estos obstáculos, ya que los distintos imaginarios que se tienen sobre el tema no posibilitan un conocimiento sobre los diferentes tipos de discapacidad. El simple hecho de ser tildado como discapacitado cierra puertas a los ámbitos de la vida. En el ámbito laboral, dependiendo de la discapacidad que la persona tenga, uno de los obstáculos puede ser la movilidad, la infraestructura de la empresa, el transporte (las personas con discapacidad física o sensorial). El desconocimiento, la comunicación entre otras cosas, para personas que tengan una discapacidad cognitiva

En cierta forma como lo ha expresado el investigadores García Núñez \& Bustos Silva (2015), retomando lo planteado por Campadabal (2001) en los siguientes términos: "la adaptación de una persona con discapacidad a su familia y a su comunidad exige un esfuerzo de ambas partes por superar la situación desventajosa que, en ciertas áreas, impide la integración del individuo para llevar una vida social normal" (p. 3). De acuerdo a esto, es claro que a nivel familiar se presentan muchos conflictos que los padres por miedo a la actitud de sus hijos o por sentimiento de culpa no aceptan o no son conscientes de ellos, las dinámicas familiares cambian; el familiar a cargo de los cuidados de la persona en situación de discapacidad deja de lado su vida personal por atender y estar pendiente de cada una de las necesidades físicas y económicas de esta persona o verse en la necesidad de contratar a otra persona para estas tareas, generando más gastos. Además, debe haber una persona a cargo de los ingresos económicos del hogar, y una no siempre es suficiente para satisfacer las demandas del núcleo familiar, en este caso casi siempre es el padre, quien debe hacer un sobre esfuerzo físico y emocional que 
afecta a la familia. Estas son las consecuencias de la exclusión laboral a personas en situación de discapacidad.

Las personas que forman parte de una familia en donde alguno de sus integrantes padece un tipo de discapacidad, son más vulnerables a situaciones de estrés, a cambios continuos en los roles dentro y fuera de la familia y una mayor exigencia de tiempo. Las familias con hijos con necesidades especiales pueden experimentar, tanto efectos positivos, por ejemplo, reforzamiento de los lazos entre la pareja, revalorización del matrimonio, aumento de la autoestima, etcétera, como negativos, por ejemplo, crisis en la pareja, ruptura de lazos matrimoniales, agresiones físicas y psicológicas en el matrimonio.

\section{Conclusiones}

La discapacidad cognitiva solo dificulta el aprendizaje y el hablar, a la persona que la padece. No afecta los ámbitos de la vida de quienes la sufren; quienes afectan directamente los ámbitos de la vida de estas personas son la sociedad con sus estigmas, la familia con su sobreprotección, las empresas con su exclusión laboral.

Las personas en situación de discapacidad sufren constantemente de estereotipos y estigmatizaciones, esta acción es frecuente en personas de su comunidad, familiares y amigos. Para algunos de ellos, esto está desprovisto de mala intención, pero los padres consideran que ello por la forma repetitiva si lo es, produciendo un rechazo en estas personas.

Hay limitaciones en la movilidad de acuerdo a la discapacidad que una persona tenga, ya sea física o sensorial; la limitación en la movilidad no es un obstáculo para la inserción laboral de las personas con discapacidad cognitiva. Las personas que presentan una discapacidad cognitiva son más frecuentes a sufrir exclusión laboral ya que es una discapacidad que no les permite comunicarse normalmente, además de eso, su proceso de aprendizaje es lento y esto es un factor de riesgo para las empresas.

Hay afectaciones económicas y sociales a nivel familiar e individual debido al estigma que se crea en la sociedad, la ignorancia y el desconocimiento que hay. Si estas 
situaciones no se presentarán, las tasas de desempleo en personas en situación de discapacidad no fueran tan preocupantes.

La desinformación que tienen los padres y los familiares que cuidan a los egresados de la Fundación CE Camilo y para esta misma se constituye en un agravante para sus condiciones económicas ya que los padres desconocen los programas y las políticas que existen en Colombia y el Distrito de Barranquilla para la inclusión laboral de las personas en condición de discapacidad

Además, el estrato socioeconómico es un factor agravante para la condición económica de las familias con personas en situación de discapacidad puesto que según las políticas del Estado, las familias de bajos estratos, son familias que tienen muy pocos ingresos económicos. Esto no es favorable para las familias, por cuanto las demandas del hogar y los cuidados de la persona en situación de discapacidad, implican tener recursos económicos. La mayoría de las personas en situación de discapacidad no cuentan con ingresos económicos suficientes, debido a que no laboran, por tal motivo no hay independencia económica y por consiguiente no hay satisfacción económica, lo cual quiere decir, que ellos no satisfacen sus necesidades por sus propios medios.

La sobreprotección que los familiares cuidadores sienten para con sus hijos no les posibilita ni les facilita el acceso a la vida laboral y por consiguiente una independencia económica que les oriente a una calidad de vida tanto a ellos como a sus padres y familiares. Es evidente que las familias se acostumbraron a mantener esta población como incapacitada, incapaz de lograr sus objetivos, de lograr una vida independiente en donde no solo puedan obtener ingresos económicos sino que también puedan tener una vida normal y realizarse como personas, tener una familia y una estabilidad emocional. La dependencia emocional es mutua. Para los padres es difícil darles autonomía a sus hijos, ya que están acostumbrados a esa dependencia, es claro que no solo hay una dependencia económica si no emocional que perjudica a la familia en general.

El apego, la dependencia emocional y el miedo es otra forma de exclusión laboral que los padres manifiestan frente a la inclusión laboral de sus hijos, puesto que es más fuerte el miedo que sienten que ver la superación en sus hijos. La exclusión laboral también es otra forma de manifestación de las desigualdades sociales que hay en el país, puesto que aun con leyes que buscan beneficiar e integrar las personas en condición de 
discapacidad al ámbito laboral, las estadísticas siguen siendo preocupantes ya que no toman en cuenta las leyes.

La Fundación CE CAMILO no brinda una formación técnica o profesional de acuerdo a las demandas que el mercado laboral impone ya que tampoco cuenta con convenios con universidades públicas o privadas para que también haya inclusión educativa de estas personas. Es importante traer a colación los Beneficios que otorga la Ley 361 de 1997 a los "particulares empleadores que vinculen laboralmente personas con limitación tendrán las siguientes garantías" (1997, p. 5):

a. A que sean preferidos en igualdad de condiciones en los procesos de licitación, adjudicación y celebración de contratos, sean estos públicos o privados si estos tiene en sus nóminas por lo menos un mínimo del $10 \%$ de sus empleados en las condiciones de discapacidad enunciadas en la presente ley debidamente certificadas por la oficina de trabajo de la respectiva zona y contratados por lo menos con anterioridad de un año; igualmente deberán mantenerse por un lapso igual al de la contratación;

b. Prelación en el otorgamiento de créditos subvenciones de organismos estatales, siempre y cuando estos se orienten al desarrollo de planes y programas que impliquen la participación activa y permanente de personas con limitación;

c. El Gobierno fijará las tasas arancelarias a la importación de maquinaria y equipo especialmente adoptados o destinados al manejo de personas con limitación. El Gobierno clasificará y definirá el tipo de equipos que se consideran cubiertos por el beneficiario (p.p. 5-6). 


\section{Referencias.}

Álvarez Ladino, A. del P.; Olaya Jaime, Z. J.; Sánchez Palacio, A. M. \& Sopó Gacharna, L. B. (2015). Aportes del Trabajo Social al concepto de inclusión socio - laboral en personas con discapacidad. Bogotá. Trabajo de Grado Universidad de la Salle. Facultad de Ciencias Económicas y Sociales. Programa de Trabajo Social. Disponible en:

http://repository.lasalle.edu.co/bitstream/handle/10185/17622/62092053_2015.pdf? sequence $=3$ \&isAllowed $=y$

Chacón Fuertes, F. (1986). Estereotipos regionales de los madrileños. Papeles del Psicólogo, Vol. (25). Consultado en octubre de 2019 en: http://www.papelesdelpsicologo.es/resumen?pii=266

Congreso de Colombia. (1997). Ley 361 de 1997. Departamento Administrativo de la Función Pública. Disponible en:

https://www.funcionpublica.gov.co/eva/gestornormativo/norma_pdf.php?i=343

De León Vargas, G. I., Ballestas León, D. M., \& Martinez Martinez, F. (2017). Amartya Sen, teoría de un desarrollo integral, aportes para la superación de la pobreza extrema y construcción de paz en los Montes de María. Erg@omnes, 9(1), 33-52. https://doi.org/10.22519/22157379.1010

Fernández Gabalón, C. (2013). La Discapacidad Intelectual en la sociedad. Percepción e integración social. Trabajo Final de Grado Educación Social. Disponible en: http://diposit.ub.edu/dspace/bitstream/2445/53928/1/Clara_Fernandez_Gabalon.pd f

http://reme.uji.es/articulos/agarce4960806100/texto.html

García Núñez, R. \& Bustos Silva, G. (2015). Discapacidad y problemática familiar, Paakat: Revista de Tecnología y Sociedad, "Nuevas tecnologías y comercio electrónico". Año 5, no. 8 (marzo-agosto 2015), pp. 1-8. Disponible en: file:///C:/Users/Rosario\%20Reales/Downloads/Dialnet-

DiscapacidadYProblematicaFamiliar-5695433.pdf

Macías Amarís, M.; Madariaga Orozco, C.; Valle Amarís, M. \& Zambrano, Javier (2013). Estrategias de afrontamiento individual y familiar frente a situaciones de estrés psicológico. Psicología desde el Caribe, 30 (1), 123-145. [fecha de Consulta 12 de Diciembre de 2019]. Disponible en: https://www.redalyc.org/articulo.oa?id=213/21328600007

Oliver, M. (1998). ¿Una sociología de la discapacidad o una sociología discapacitada? Disponible en: http://bivipas.unal.edu.co/bitstream/10720/645/1/262Sociologia_Discapacidad_Sociologia_Discapacitada_Capitulo_2-Oliver_Mike.pdf 
Organización Mundial de la Salud. (2011). Informe mundial sobre la discapacidad 2011. Disponible en: http://www1.paho.org/arg/images/Gallery/Informe_spa.pdf

Padilla-Muñoz, A. (2010). Discapacidad: contexto, concepto y modelos. Revista Colombiana de Derecho Internacional, (2010), pp. 381-414. Disponible en: http://www.scielo.org.co/pdf/ilrdi/n16/n16a12.pdf 